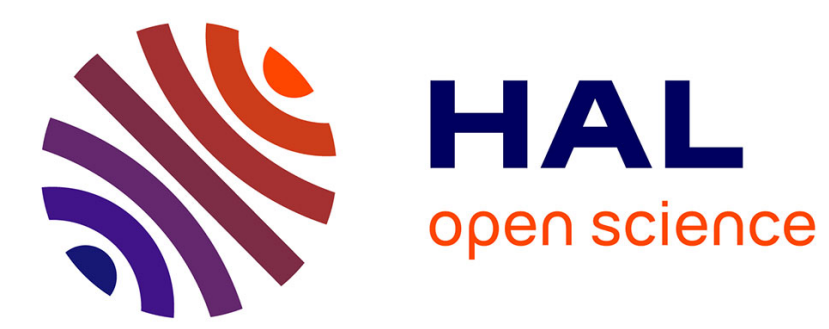

\title{
Using column generation to compute lower bound sets for bi-objective combinatorial optimization problems
}

\author{
Boadu Mensah Sarpong, Christian Artigues, Nicolas Jozefowiez
}

\section{To cite this version:}

Boadu Mensah Sarpong, Christian Artigues, Nicolas Jozefowiez. Using column generation to compute lower bound sets for bi-objective combinatorial optimization problems. RAIRO - Operations Research, 2015, 49 (3), pp.527 - 554. 10.1051/ro/2014054 . hal-01875890

\section{HAL Id: hal-01875890 \\ https://hal.laas.fr/hal-01875890}

Submitted on 15 Sep 2021

HAL is a multi-disciplinary open access archive for the deposit and dissemination of scientific research documents, whether they are published or not. The documents may come from teaching and research institutions in France or abroad, or from public or private research centers.
L'archive ouverte pluridisciplinaire HAL, est destinée au dépôt et à la diffusion de documents scientifiques de niveau recherche, publiés ou non, émanant des établissements d'enseignement et de recherche français ou étrangers, des laboratoires publics ou privés. 


\title{
Using Column Generation to Compute Lower Bound Sets for Bi-Objective Combinatorial Optimization Problems
}

\author{
Boadu Mensah Sarpong, ${ }^{1,2}$ Christian Artigues ${ }^{1,3}$, Nicolas Jozefowiez ${ }^{1,3}$ \\ ${ }^{1}$ CNRS, LAAS, 7 avenue du colonel Roche, F-31400 Toulouse, \\ France \\ ${ }^{2}$ Univ de Toulouse, INSA, LAAS, F-31400 Toulouse, France \\ ${ }^{3}$ Univ de Toulouse, LAAS, F-31400 Toulouse, France
}

\begin{abstract}
We discuss the use of column generation in a bi-objective setting. Just as in single objective combinatorial optimization, the role of column generation in the bi-objective setting is to compute dual bounds (i.e. lower bounds for minimization problems and upper bounds for maximization problems) which can be used to guide the search for efficient solutions or to evaluate the quality of approximate solutions. The general idea used in this paper is to first transform the bi-objective problem into single objective by a scalarization method and then solve the transformed problem several times by varying the necessary parameters. We show that irrespective of the scalarization method used, similar subproblems are solved when applying column generation. For this reason, we investigate possible ways of intelligently searching for columns for these subproblems in order to accelerate the column generation method.
\end{abstract}

keywords Multi-Objective Optimization, Bound Sets, Combinatorial Optimization, Column Generation

\section{Introduction}

Exact methods for solving difficult single objective optimization problems rely heavily on lower and upper bounds. The number of exact methods for multiobjective problems is very few and a possible explanation for this is that the notion of bounds is not very well developed for multi-objective problems in comparison to single objective problems. For this reason, it is necessary to develop models and strategies for computing good bounds for multi-objective problems and this paper aims at contributing to this goal. Without loss of generality, we suppose that all objective functions of the considered problems are to be minimized. We study the use of column generation in computing lower 
bound sets for bi-objective combinatorial optimization (BOCO) problems. This study is expected to serve a broad range of applications since an important class of combinatorial optimization problems can be formulated as integer programs with exponential number of variables that are efficiently solved by column generation methods. An advantage of this type of formulations is that they usually have good linear relaxations and thus strong lower bounds.

\subsection{Multi-Objective Combinatorial Optimization}

A general multi-objective combinatorial optimization (MOCO) problem deals with the minimization of a vector of two or more functions $F(x)=\left(f_{1}(x), \ldots, f_{r}(x)\right)$ over a finite domain of feasible solutions $\mathcal{X}$. The vector $x=\left(x_{1}, \ldots, x_{n}\right)$ is the decision variable or solution, $\mathcal{Y}=F(\mathcal{X})$ corresponds to the images of the feasible solutions in the objective space, and $y=\left(y_{1}, \ldots, y_{r}\right)$, where $y_{i}=f_{i}(x)$, is a point of the objective space. A solution $x^{\prime}$ dominates another solution $x^{\prime \prime}$, (denoted $\left.x^{\prime} \preceq x^{\prime \prime}\right)$, if for any index $i \in\{1, \ldots, n\}, f_{i}\left(x^{\prime}\right) \leq f_{i}\left(x^{\prime \prime}\right)$ and there is at least one index $i \in\{1, \ldots, n\}$ for which $f_{i}\left(x^{\prime}\right)<f_{i}\left(x^{\prime \prime}\right)$. A feasible solution dominated by no other feasible solution is said to be efficient or Pareto optimal and its image in the objective space is said to be nondominated. The set of all efficient solutions is called the efficient set (denoted $\mathcal{X}_{E}$ ) and the set of all nondominated points is the nondominated set (denoted $\mathcal{Y}_{N}$ ). In general, more than one efficient solution may correspond to the same nondominated point and so solving a MOCO problem in most practical cases usually means finding at least one efficient solution for each nondominated point. The nondominated set defines what is known as the Pareto frontier. An efficient solution that maps onto a nondominated point lying on the convex part of the Pareto frontier is called a supported efficient solution and its image is called a supported nondominated point. An efficient solution that is not supported is called a nonsupported efficient solution.

\subsubsection{Lower and Upper Bounds for a MOCO Problem}

Although the meaning of bounds in single objective optimization is well studied and understood, the situation is quite different in the multi-objective case. Ideal and nadir points are well known lower and upper bounds, respectively, of the set $\mathcal{Y}_{N}$. The coordinates of the ideal point are obtained by optimizing each objective function independently of the others, whereas the coordinates of the nadir point correspond to the worst value of each objective function when we consider the set $\mathcal{X}_{E}$. From Figure ??, it can be seen that these points are usually poor bounds since they just estimate the whole region where a member of $\mathcal{Y}_{N}$ may lie. In this paper, we will be interested in bounds that can reduce the region where the members of $\mathcal{Y}_{N}$ are and thus narrow down the search for nondominated points.

Given that a MOCO problem is a discrete problem, its lower bound can be defined as a set of points $\boldsymbol{L}$ such that the image of every feasible solution is either a member of $\boldsymbol{L}$ or is dominated by at least one member of $\boldsymbol{L}$. The members of a lower bound set do not necessarily belong to $\mathcal{Y}$. That is, they may or may not correspond to feasible points of the problem. An upper bound 


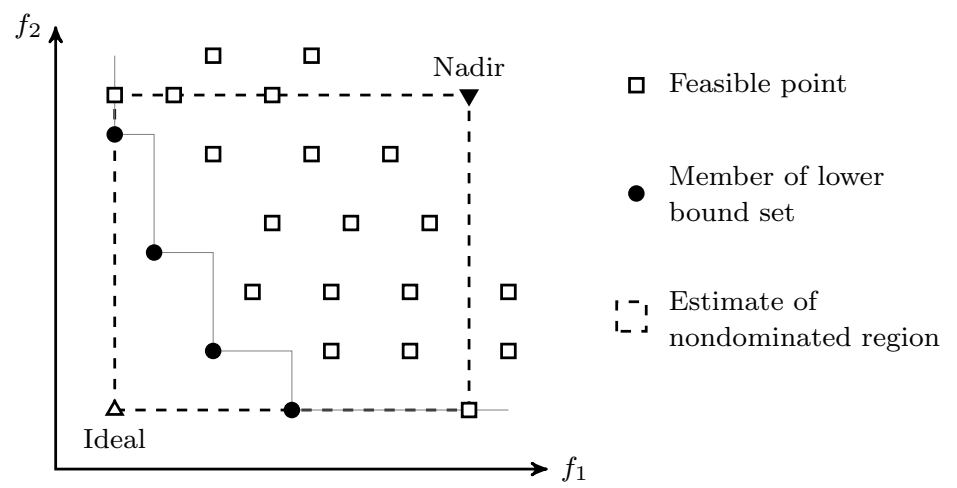

Figure 1: Lower and upper bounds for a BOCO problem.

may also be defined as a finite set of points in $\mathcal{Y}$ that do not dominate one another. The idea of using sets to define bounds for multi-objective problems was first introduced in [?]. The constuction and use of bound sets for bi-objective combinatorial optimization (BOCO) problems has recently been addressed by some authors [?, ?, ?]. The main idea used by these authors in defining a lower bound set is to transform the bi-objective problem into single objective by using a weighted sum method and solving the transformed problem for different weights in order to compute the complete set of supported nondominated points. A lower bound set for the considered bi-objective problem may be defined as the line connecting the set of supported nondominated points. This procedure is only possible if there is an efficient algorithm for solving the single objective problem associated to the bi-objective problem. If the single objective problem associated to the bi-objective problem is $\mathcal{N} \mathcal{P}$-hard, then we have to resort to computing the set of supported nondominated points for a relaxation of the single objective problem. As pointed out in [?], any scalarization method may be used to convert a bi-objective problem into single objective when computing bound sets. Nevertheless, we could not find a published paper that uses a method not based on the weighted sum scalarization. One reason for this may be that the weighted sum method converts a multi-objective problem into single objective by changing only the objective function. In this way, well known single objective methods can be used to solve the transformed problem. A well known disadvantage of the weighted sum method is that it cannot find nonsupported solutions no matter the choice of weights used. If there is a large number of nonsupported points, a lower bound set produced by using a weighted sum method can be very poor. For this reason, it seems worthy to study the use of another scalarization method like the $\varepsilon$-constraint method in computing bound sets. A disadvantage of an $\varepsilon$-constraint method is that it is not very easy to define values for the parameter $\varepsilon$ in such a way that all members of the nondominated set are found. Nevertheless, this is possible for some practical problems (see for example [?, ?]). An advantage of the $\varepsilon$-constraint method is that, unlike a weighted sum method, it is capable of finding both supported and nonsupported 
solutions.

An important class of combinatorial optimization problems (e.g. vehicle routing problems) can be formulated as (mixed-)integer linear programs with an exponential number of variables. These type of formulations (set covering, and set packing models) are efficiently solved by column generation based methods. In spite of the challenge posed by the large number of variables, an advantage of this type of models is that they usually have strong linear relaxations and so produce good lower bounds. In this paper, we discuss how column generation can be used to compute lower and upper bound sets for BOCO problems which are modeled by an exponential number of variables. In particular, we show that a set of similar subproblems are solved whether we use a weighted sum method or an $\varepsilon$-constraint method. For this reason, we investigate the possibility of treating some of these subproblems simultaneously in order to speed up the column generation algorithm.

\subsection{Contributions and Organization of Work}

A first contribution of this work is the application of column generation to BOCO problems. Although column generation and multi-objective optimization are well studied independently, the application of column generation to multi-objective problems seems to have been neglected. This current paper contributes in this respect. In particular, we propose different approaches to effectively search for columns when applying column generation to BOCO problems. Another contribution of this work is in the use of a scalarization method, different from the weighted sum, in computing lower bound sets for BOCO problems. To the best of our knowledge, this is the first attempt at designing such a method.

In Section ??, we discuss how column generation can be used in computing bound sets for BOCO problems. We show that whether we use a weighted sum method or an $\varepsilon$-constraint method, a similar type of subproblem is encountered. Due to this, we propose different strategies to effectively search for relevant columns. An application problem is presented in Section ?? and evaluation of the bound sets computed for this problem as well as a comparison of the different column search strategies are given in Section ??. In section ??, we draw some conclusions and point out possible ways of extending this work.

\section{Column Generation for BOCO Problems}

In this section, we discuss how column generation can be used to compute lower and upper bound sets for BOCO problems by considering the following bi-objective set-covering based formulation, $P$ :

$$
\begin{aligned}
\text { Minimize } & \left(c^{1}\right)^{T} x \\
\text { Minimize } & \left(c^{2}\right)^{T} x \\
\text { subject to : } & A x \geq b, \\
& x \geq 0 \text { and integer. }
\end{aligned}
$$


In the above formulation, $x$ is a vector of $n$ decision variables and $c^{i}$ is a vector of $n$ integer coefficients in the $i$ th objective function $(i=1,2)$. The constraints are expressed by using an $m \times n$ matrix of coefficients, $A$, and a vector of $m$ constants, $b$.

\subsection{Computing Lower and Upper Bound Sets}

The main idea used in computing bound sets for BOCO problems is to convert the problem into single objective by using a scalarization method and solving the resulting problem (or a relaxation of it) several times by varying the necessary parameters. In this paper, we consider the $\varepsilon$-constraint method and the weighted sum method.

The weighted sum method transforms Formulation (??-??) into single objective by using a vector of non-negative weights $\lambda=\left(\lambda_{1}, \lambda_{2}\right)$. The resulting problem $P(\lambda)$ is given by

$$
\begin{aligned}
\text { Minimize } & \lambda_{1} \cdot\left(c^{1}\right)^{T} x+\lambda_{2} \cdot\left(c^{2}\right)^{T} x \\
\text { subject to : } & A x \geq b, \\
& x \geq 0 \text { and integer. }
\end{aligned}
$$

On the other hand, the $\varepsilon$-constraint approach obtains a single objective problem by restricting the worst possible value of one objective (say the second one) to a constant $\varepsilon \in \mathbb{R}$. Thus, we obtain the single objective problem $P(\varepsilon)$ :

$$
\begin{array}{rrr}
\text { Minimize } & \left(c^{1}\right)^{T} x \\
\text { subject to : } & A x & \geq b \\
-\left(c^{2}\right)^{T} x & \geq-\varepsilon \\
& x \geq 0 \text { and integer. }
\end{array}
$$

In a first instance, we suppose that Formulation (??-??) is manageable in the sense that we have a "small" number of variables in $x$ (i.e. $n$ is small). In such a case, there is no need for column generation in computing a lower bound set for problem $P$. This has been discussed extensively in [?] for the weighted sum method. Given that Formulation (??-??) is $\mathcal{N} \mathcal{P}$-hard in the general case, we need to find the set of supported nondominated points for a relaxation of it. We consider the linear relaxation which is obtained by dropping the requirement for $x$ to be integer. The set of supported nondominated points can be obtained by a method proposed in [?] which is used widely as the first phase of the two phases method [?]. A lower bound set can then be defined by the line joining the points of the computed set.

\subsubsection{Using an $\varepsilon$-Constraint Method}

The main idea in using an $\varepsilon$-constraint method to compute lower bound sets is similar to the case where we use a weighted sum method. That is, we need to solve linear relaxations of $P(\varepsilon)$ for different values of $\varepsilon$. In doing so, we need 


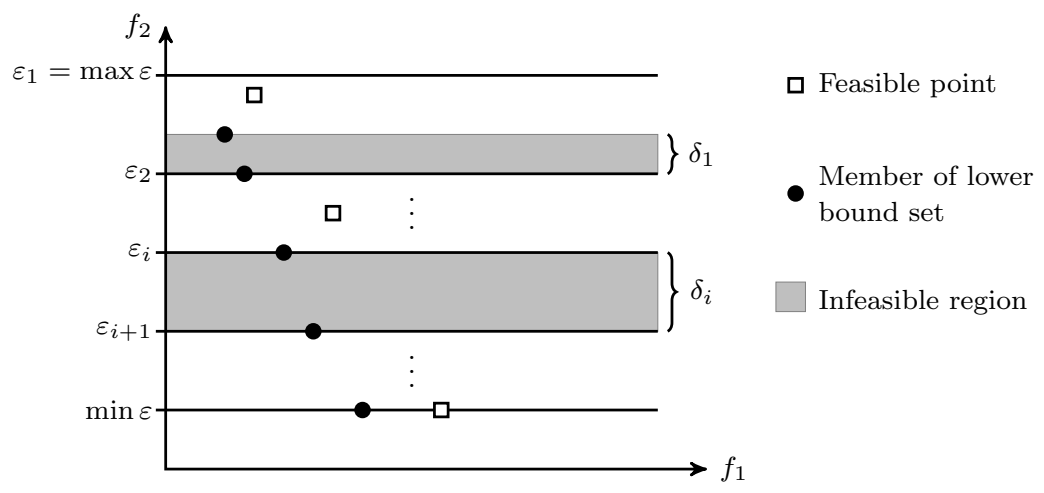

Figure 2: Constructing a lower bound set through an $\varepsilon$-constraint method.

to ensure that every feasible point of the problem $P$ is either generated or is dominated by at least one of the generated points. The general procedure is summarized in Algorithm ?? and we suppose that all feasible values for $\left(c^{2}\right)^{T} x$ lie in the range $[\min \varepsilon, \max \varepsilon]$. The output of Algorithm ?? is the set $\boldsymbol{L}$ and a sequence of values $\max \varepsilon=\varepsilon_{1}>\varepsilon_{2}>\cdots>\min \varepsilon$ corresponding to the elements of the set. Note that each step size $\delta_{i}$ is chosen in such a way that there can be no feasible point $\left(f_{1}^{*}=\left(c^{1}\right)^{T} x^{*}, f_{2}^{*}=\left(c^{2}\right)^{T} x^{*}\right)$ of the original integer program such that $\varepsilon_{i+1}<f_{2}^{*}<f_{2}^{i}$ (see Figure ??). This is a very difficult thing to do for a general bi-objective problem. For the considered problem, however, we have integer objective coefficients so we can use an idea similar to the one used in [?] and which was formalized in [?]. At iteration $i$, we define the step size as $\delta_{i}=1-\left(\left\lceil f_{2}^{i}\right\rceil-f_{2}^{i}\right)$. As we will later see, it is possible to define even better step sizes for specific problems. It can be clearly seen that the set, $\boldsymbol{L}$, returned by the algorithm is indeed a lower bound set for the considered problem.

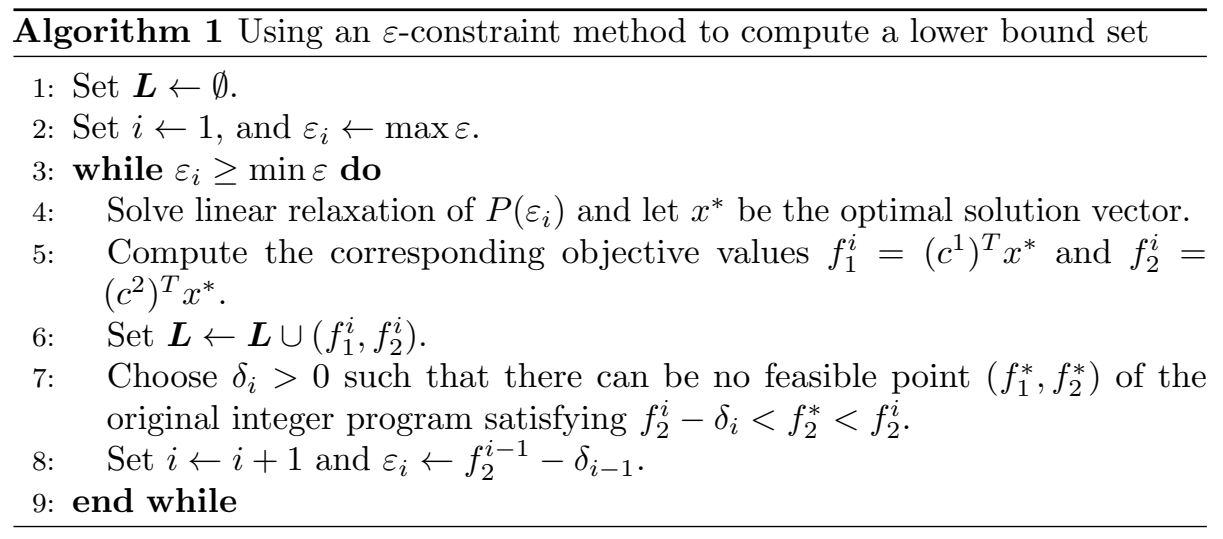

Whether we use a weighted sum method or an $\varepsilon$-constraint method, the members of a lower bound set may correspond to points that are infeasible for 
problem $P$. The definition of an upper bound set, however, requires that its members are feasible points of $P$. For this reason, heuristics and metaheuristics are used in computing upper bound sets when necessary. An example is the greedy heuristic used in computing upper bound sets for instances of the biobjective set covering problem in [?]. We stress that the heuristic or metaheuristic used in computing an upper bound set does not necessarily need to depend on a particular scalarization method. Any known heuristic or metaheuristic for problem $P$ may be used.

\subsection{Computing Lower Bound Sets by Column Generation}

Many practical problems can be formulated in the form of (??-??) with an exponential number of decision variables in order to obtain a tight linear programming relaxation. It is impractical and sometimes impossible to consider all the variables at once when dealing with such formulations. The idea of a column generation method for solving such a problem is to start with only a reasonable number of variables (and the corresponding columns of matrix $A$ ) for which the problem is primal feasible. The other variables together with their corresponding columns of $A$ are introduced when necessary by solving an auxiliary problem. In column generation terminology, the original integer problem with an exponential number of variables (or columns) is called the integer programming master problem (IPM) and its linear relaxation is called the linear programming master problem (LPM). A restriction of IPM (respectively, LPM) to a reasonable number of columns is called a restricted IMP (RIPM), respectively, RLPM. The auxiliary problem solved to propose new variables to add to the RLPM or prove the convergence of the method is called the subproblem $(S)$. Each iteration of column generation starts by solving the RLPM in order to obtain optimal primal and dual solutions. The subproblem $(S)$ uses the optimal dual values to search for new columns that can possibly improve the current objective value of the RLPM. One or several of such columns, if found, are added to the RLPM and the process repeats. The method converges when the subproblem finds no new columns that can possibly improve the current objective value of the RLPM.

Next, we discuss how to use column generation in computing lower bounds for BOCO problems modelled with an exponential number of variables. We list the different models involved in a column generation method in the case of a weighted sum method and also those in the case of an $\varepsilon$-constraint method.

\subsubsection{Using a Weighted Sum Method}

Linear Programming Master Problem $(\operatorname{LPM}(\lambda))$ :

$$
\begin{aligned}
\text { Minimize } & \left(\lambda_{1} c^{1}+\lambda_{2} c^{2}\right)^{T} x \\
\text { subject to : } & A x \geq b \\
& x \geq 0 .
\end{aligned}
$$


Dual of LPM $(\operatorname{DLPM}(\lambda))$ : Let $\pi$ be the vector of dual variables associated with Constraints (??). The dual formulation is

$$
\begin{aligned}
\text { Maximize } & b^{T} \pi \\
\text { subject to : } & A^{T} \pi \leq \lambda_{1} c^{1}+\lambda_{2} c^{2}, \\
& \pi \geq 0 .
\end{aligned}
$$

Subproblem $(\mathrm{S}(\lambda))$ : It is defined as finding variables that correspond to columns of matrix $A$ and which satisfy an equality of the form

$$
\lambda_{1} c_{j}^{1}+\lambda_{2} c_{j}^{2}-A_{j}^{T} \pi<0
$$

where $A_{j}$ is the $j$-th column of matrix $A$.

\subsubsection{Using an $\varepsilon$-Constraint Method}

Linear Programming Master Problem $(\operatorname{LPM}(\varepsilon))$ :

$$
\begin{aligned}
\text { Minimize } \quad\left(c^{1}\right)^{T} x & \\
\text { subject to }: A x & \geq b, \\
\left(-c^{2}\right)^{T} x & \geq-\varepsilon, \\
x & \geq 0 .
\end{aligned}
$$

Dual of LPM $(\operatorname{DLPM}(\varepsilon))$ : Let $\pi$ be the vector of dual variables associated with Constraints (??) and $\varphi$ the dual variable associated with Constraint (??). The dual is formulation is

$$
\begin{aligned}
\text { Maximize } & b^{T} \pi-\varepsilon \varphi \\
\text { subject to : } \quad A^{T} \pi-\varphi c^{2} & \leq c^{1}, \\
\pi, \varphi & \geq 0 .
\end{aligned}
$$

Subproblem $(\mathrm{S}(\varepsilon))$ : It is defined as finding variables that correspond to columns of matrix $A$ and which satisfy an equality of the form

$$
c_{j}^{1}+\varphi c_{j}^{2}-A_{j}^{T} \pi<0,
$$

where $A_{j}$ is the $j$-th column of matrix $A$.

\subsubsection{General Form of the Subproblem}

By comparing the inequalities (??) and (??), we can see that the subproblems encountered in both cases have a similar form. In addition, the general form of the subproblem obtained by using a particular scalarization method does not change when we modify the parameters. Only the values of the dual variables and coefficients change. A similar result is obtained if we consider problems with more than two objectives. This means that strategies we describe in solving 
the subproblems obtained by one of these scalarization methods can easily be adapted to the other scalarization methods. Moreover, for any given scalarization method, it is possible to treat more than one subproblem at the same time when searching for columns. We present different strategies to search for column in Section ??.

\subsubsection{Computing an Upper Bound Set}

A direct and intuitive way of computing an upper bound set after computing a lower bound set by column generation is to solve the RLPM (with its current columns) as an integer program several times by following the idea of Algorithm ??. Before applying this algorithm, it is possible to use knowledge of the problem in order to generate more columns that were not necessary in computing a lower bound set but may be useful when solving the RLPM as an integer program. It is also possible to use metaheuristics and other problem specific heuristics if they are available.

\subsection{Column Search Strategies}

As we have already noted, the general form of subproblem obtained by using a particular scalarization method does not change when we modify the parameters. Also, irrespective of the scalarization method used, the subproblem has a similar form. We discuss some approaches to search for relevant columns when computing a lower bound set by column generation. We will demonstrate the strategies for the case where we use an $\varepsilon$-constraint method. They can, however, be easily adapted to the case where a weighted sum method is used. In the case where a particular idea applies uniquely to an $\varepsilon$-constraint method, this will be stated.

\subsubsection{Point-by-Point Search (PPS)}

A standard and very intuitive approach to apply column generation to a BOCO problem involves solving the RLPM completely for any given value of $\varepsilon$ before continuing to the next value of $\varepsilon$. That is, for any given value of $\varepsilon, \operatorname{RLPM}(\varepsilon)$ is solved by column generation until the subproblem proposes no new columns that can improve the current objective value of the RLPM. We call this approach the point-by-point search (PPS) since the search is completely dedicated to finding a particular point of a lower bound set (corresponding to a particular value of $\varepsilon)$ before moving on to another point. The algorithm for the PPS is similar to the one described in Algorithm ?? except that in Step ?? we need to solve an RLPM to optimality by column generation. The columns generated while solving the RLPM for a given value of $\varepsilon$ are kept in the model throughout the whole algorithm. This is because they may still be relevant for other values of $\varepsilon$ due to the similar structures of the subproblems. The PPS identifies the points of a lower bound set following a predictable order. The point corresponding to a smaller value of $\varepsilon$ can be found only after all the points corresponding to greater values of $\varepsilon$ have been found. Although the PPS is simple and easy to 
implement, it takes no advantage of the similar form of the subproblems solved for the different values of $\varepsilon$. The column generation method may also be slow to converge for a given value of $\varepsilon$ but the PPS requires us to wait for it to converge before moving on to another value of $\varepsilon$. This can result in a huge number of "irrelevant" columns being added to the RLPM and a long computational time. Two approaches which aim at addressing some of these problems are discussed below.

\subsection{2 $\quad k$-Step Point-by-Point Search ( $k$-PPS)}

In this approach, we have the freedom to leave a given value of $\varepsilon$ before the RLPM has converged for it and switch to another value of $\varepsilon$. If it is necessary, we will return to a previously skipped value of $\varepsilon$ later on. The $k$-PPS approach is summarized in Algorithm ??. The first step of this approach is to define a condition, $\mathcal{O}$, under which a given value of $\varepsilon$ for which the RLPM has not converged will be skipped either temporarily or permanently. The condition can be as simple as setting a fixed number, $k$, of column generation iterations. Instead of using a fixed number of iterations, we may also decide to skip a given value of $\varepsilon$ when the objective value of the RLPM has not improved significantly after $k$ iterations. This can be a way of addressing some column generation problems like the plateau effect and the tailing-off effect that were described in [?]. It is also possible to use any other condition we find appropriate for a given problem. In Step ?? of Algorithm ??, there is no need to solve $\operatorname{RLPM}\left(\varepsilon_{i}\right)$ if either of these two conditions are satisfied:

1. There is a converged point $\left(f_{1}^{*}, f_{2}^{*}\right) \in \boldsymbol{L}$ such that $f_{2}^{*} \leq \varepsilon_{i} \leq \varepsilon_{*}$, where $\varepsilon_{*}$ denotes the value of $\varepsilon$ for which $\left(f_{1}^{*}, f_{2}^{*}\right)$ was computed.

2. There is a converged point $\left(f_{1}^{*}, f_{2}^{*}\right) \in \boldsymbol{L}$ and another point $\left(f_{1}^{j}, f_{2}^{j}\right)$ such that $f_{1}^{j}-f_{1}^{*} \leq 1$ and $f_{2}^{j}<\varepsilon_{i}<f_{2}^{*}$. Note that the point $\left(f_{1}^{j}, f_{2}^{j}\right)$ may have converged or not.

If the first condition is satisfied, there is no need to solve $\operatorname{RLPM}\left(\varepsilon_{i}\right)$ since we are sure to end up with a solution that maps onto $\left(f_{1}^{*}, f_{2}^{*}\right)$. In the case of the second condition, the new point that will be generated by solving $\operatorname{RLPM}\left(\varepsilon_{i}\right)$ to optimality will not make the current lower bound set any better since we have integral objective coefficients.

Unlike in the case of the PPS, the points of a lower bound set are identified by the $k$-PPS in no predictable order. That is, the RLPM can converge for a smaller value of $\varepsilon$ before it converges for a greater value. Figure ?? illustrates a possible order in which the points of a lower bound set are identified by the $k$-PPS. This illustration is based on a fictitious example. We suppose that feasible values for the $\varepsilon$-constraint objective function are integers between $\min \varepsilon=1$ and $\max \varepsilon=10$. Each complete iteration of the $k$-PPS approach will thus start by solving $\operatorname{RLPM}(\varepsilon)$ for $\varepsilon=10$ as in Figure ??. A description of the series of diagrams is given below. Note that we can obtain non-integral points since we are solving linear relaxations rather than integer programs. During the process, converged points are saved in the lower bound set. 
$\mathbf{O} i \quad i^{\text {th }}$ unconverged point generated at current iteration

- $i \quad i^{\text {th }}$ converged point from the start of the algorithm

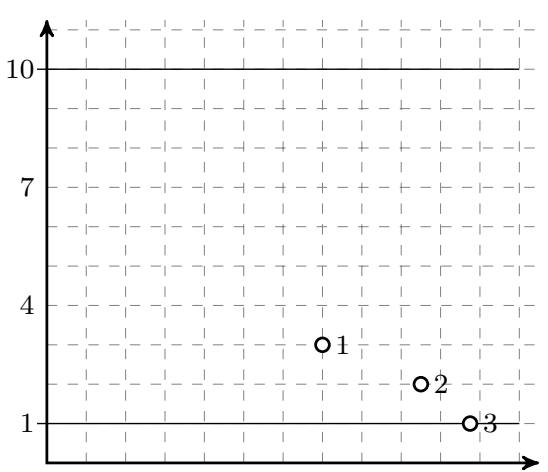

(a)

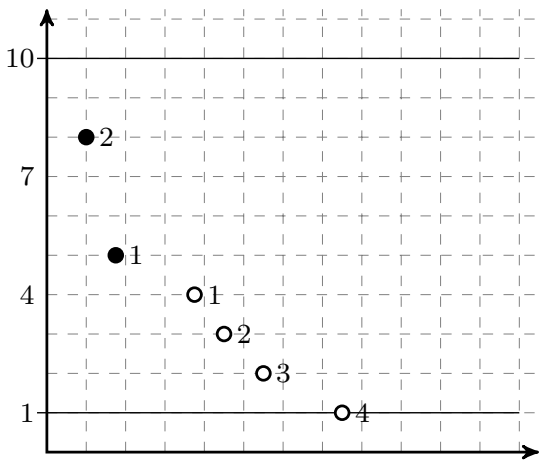

(c)

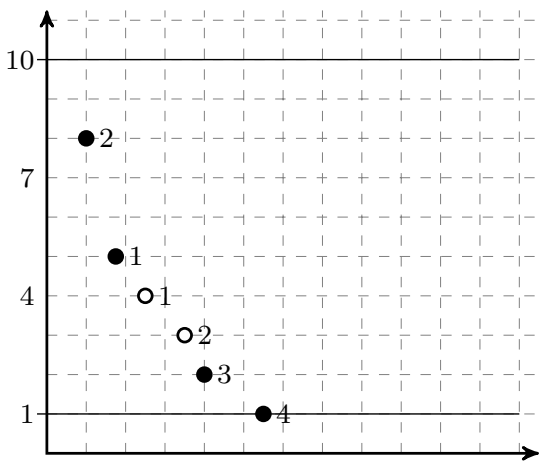

(e)

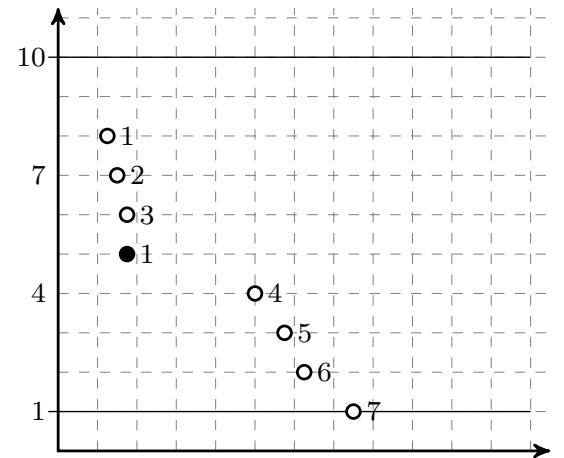

(b)

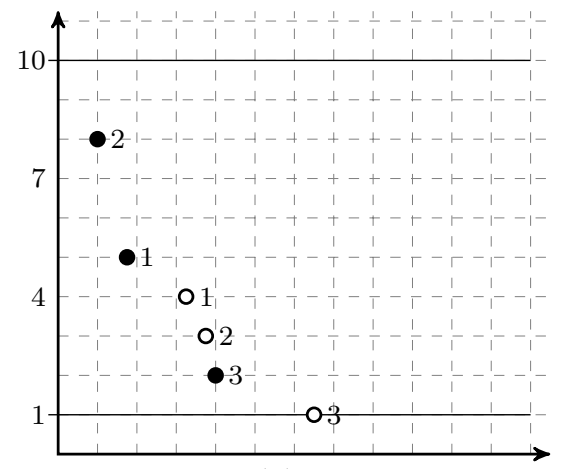

(d)

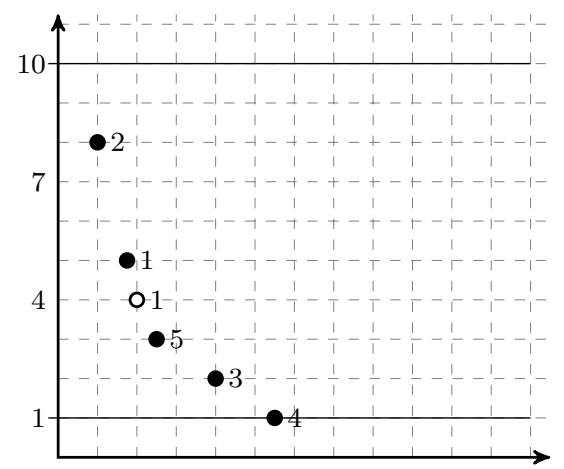

(f)

Figure 3: Possible order in which points of a lower bound set are identified by the $k$-PPS. 


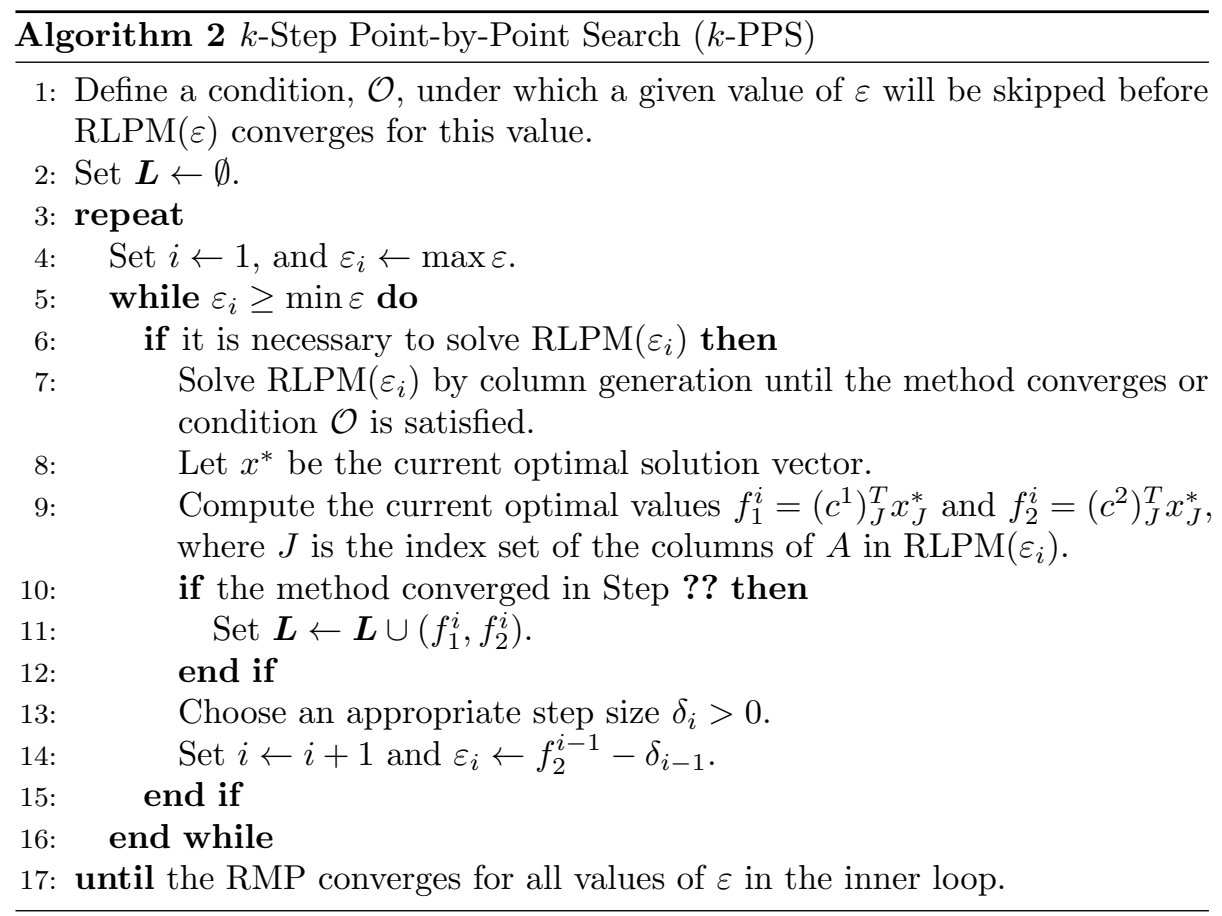

Figure ??. There are no converged points at the start of the algorithm. Condition $\mathcal{O}$ is satisfied before RLPM(10) converges and the current unconverged point is (7.0,3.0). Similarly, condition $\mathcal{O}$ is satisfied before $\operatorname{RLPM}(2)$ and $\operatorname{RLPM}(1)$ converge. The corresponding unconverged points are $(9.5,2.0)$ and $(10.75,1.0)$, respectively.

Figure ??. This iteration of $k$-PPS also starts with no converged points from the previous iteration. Condition $\mathcal{O}$ is satisfied before $\operatorname{RLPM}(10), \operatorname{RLPM}(7)$, and $\operatorname{RLPM}(6)$ converge. The corresponding points are $(1.25,8.0),(1.5,7.0)$, and $(1.75,6.0)$, respectively. $\operatorname{RLPM}(5)$ converges to the point $(1.75,5.0)$ before condition $\mathcal{O}$ is satisfied. Four more unconverged points $(5.0,4.0),(5.75,3.0)$, $(6.25,2.0)$, and $(7.5,1.0)$ corresponding to $\operatorname{RLPM}(4), \operatorname{RLPM}(3), \operatorname{RLPM}(2)$, and RLPM(1), respectively, are generated in this iteration.

Figure ??. RLPM(10) converges to the point $(1.0,8.0)$ and becomes the second converged point since the start of the algorithm. RLPM(7) and RLPM(6) are permanently skipped for the rest of the algorithm since these values of $\varepsilon$ satisfy the second condition for skipping a value of $\varepsilon$. Four unconverged points $(3.75,4.0),(4.5,3.0),(5.5,2.0)$, and $(7.5,1.0)$ corresponding to $\operatorname{RLPM}(4)$, $\operatorname{RLPM}(3), \operatorname{RLPM}(2)$, and $\operatorname{RLPM}(1)$, respectively, are generated in this iteration. 
Figure ??. There are two previously converged points at the start of this iteration. RLPM(10), RLPM(9), and RLPM(8) are skipped permanently for rest of the algorithm based on the first and second conditions for skipping a value of $\varepsilon$. Condition $\mathcal{O}$ is satisfied before $\operatorname{RLPM}(4)$ and $\operatorname{RLPM}(3)$ converge and the corresponding points are $(3.25,4.0)$ and $(3.75,3.0)$, respectively. One converged point $(4.0,2.0)$ corresponding to $\operatorname{RLPM}(2)$ is generated whereas an unconverged point $\mathrm{RLPM}(1)$ corresponding to $(6.5,1.0)$ is also produced.

Figure ??. There are three previously converged points at the start of this iteration. The values of $\varepsilon$ which were skipped in previous iterations are also skipped here for the same reasons as before. Two unconverged points $(2.5,4.0)$ and $(3.5,3.0)$ corresponding to $\operatorname{RLPM}(4)$ and $\operatorname{RLPM}(3)$, respectively, are generated. RLPM(1) converges to the point $(5.5,1.0)$.

Figure ??. This iteration starts with $\operatorname{RLPM}(4)$ and which fails to converge before condition $\mathcal{O}$ is satisfied. The corresponding unconverged point is $(2.0,4.0)$. $\operatorname{RLPM}(3)$ converges to the point $(2.5,3.0)$. There is no need for an additional iteration to return to RLPM(4) thanks to the second condition for skipping a value of $\varepsilon$.

It is important to note that the set returned by the $k$-PPS represents a lower bound for the considered BOIP. In other words, if a given value of $\varepsilon$ is skipped, then the algorithm will either return to the skipped value later on or prove that it is unnecessary to do so. Hence, no relevant points are missed. One main challenge of the $k$-PPS is that it is not easy to decide on a good condition $\mathcal{O}$ when we don't have enough information on how the RLPM behaves at the start of the algorithm. For this reason, it may be necessary to have an adaptive condition $\mathcal{O}$ that can be modified across the iterations when we have enough information.

\subsubsection{Sequential Search}

The main idea behind the sequential search approach is to work on a whole frontier rather than deal with individual points. This is achieved by returning a set of columns that are relevant for several values of $\varepsilon$ at each iteration of column generation. A description of the approach is given in Algorithm ??. Each iteration of the sequential search approach consists of two main steps. In step I (steps ?? to ?? of Algorithm ??), we solve the RLPM (without generating any columns) for several values of $\varepsilon$ for which it has not converged. The search for relevant columns is done in step II (steps ?? to ?? of Algorithm ??). During an iteration, the sets $\Pi$ and $\Omega$ are used to temporary store the vectors of dual values and the generated columns, respectively. Before solving the subproblem corresponding to a given point generated in step $I$, we first need to verify if any of the columns already found for the other points are relevant (has a negative reduced cost) for this new point. If this is the case the new point is skipped without solving the subproblem corresponding to it since the point is already represented by the set of generated columns. Thus, the subproblem 
corresponding to a new point is only solved if there are no previously found columns in the same iteration that are relevant for this point.

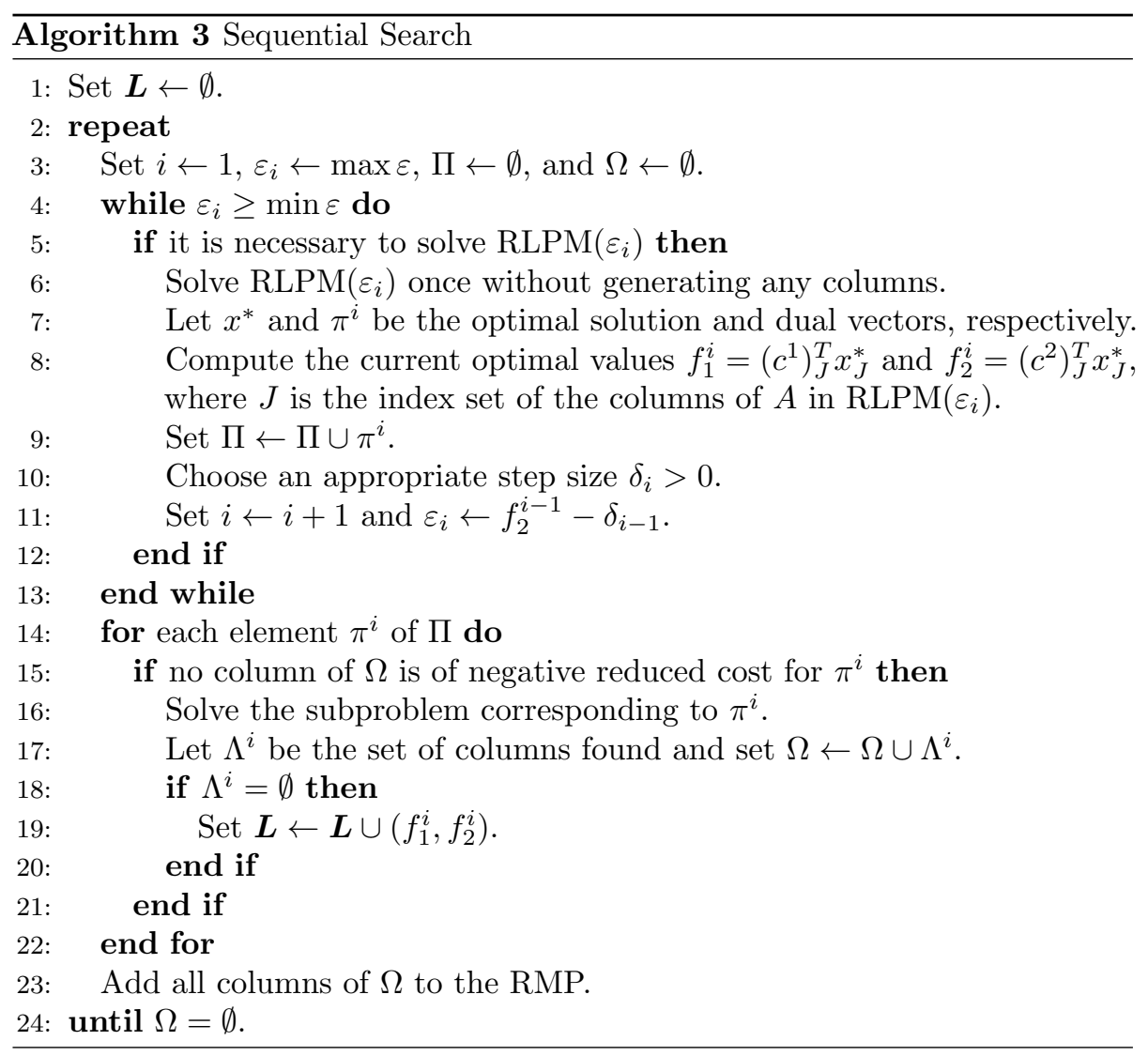

There is no particular order in which the points of a lower bound set are identified by a sequential search method. Note, however, that a point generated in step $I$ of an iteration can be proven to have converged in step $I I$ only if the subproblem corresponding to it is solved directly by an exact algorithm and no relevant columns are found. A fictitious example to demonstrate a possible order in which the points of a lower bound set are identified by a sequential search approach is given in the series of diagrams in Figure ??. An explanation of each of the diagrams is given below.

Figure ??. There are no converged points at the start of the algorithm. Step $I$ produces three points $(7.0,3.0),(9.5,2.0)$ and $(21.5,1.0)$ corresponding to $\operatorname{RLPM}(10), \operatorname{RLPM}(2)$, and $\operatorname{RLPM}(1)$, respectively. None of these points is proven to have converged in step $I I$. 
$\mathbf{O} i \quad i^{\text {th }}$ unconverged point generated at current iteration

- $i \quad i^{\text {th }}$ converged point from the start of the algorithm

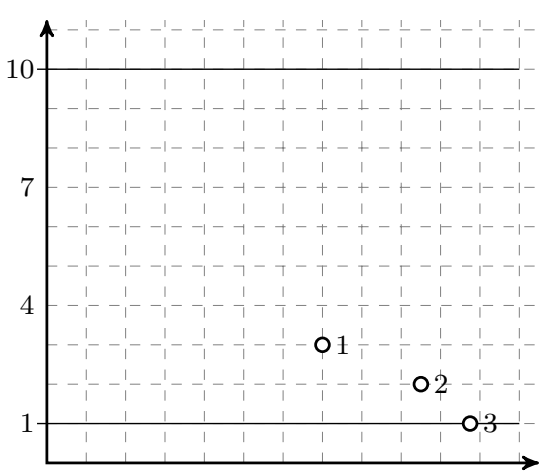

(a)

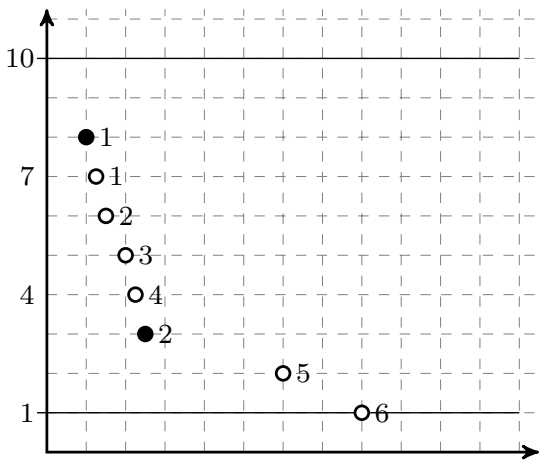

(c)

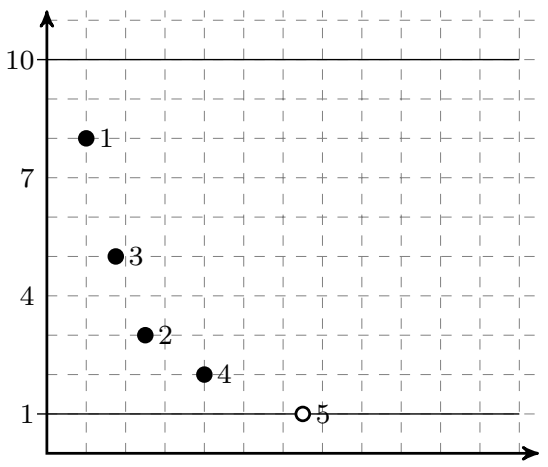

(e)

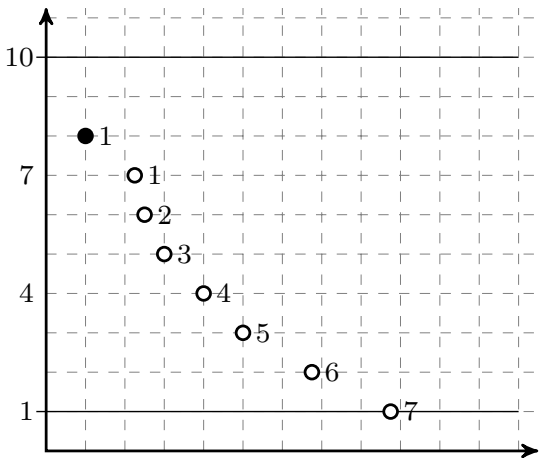

(b)

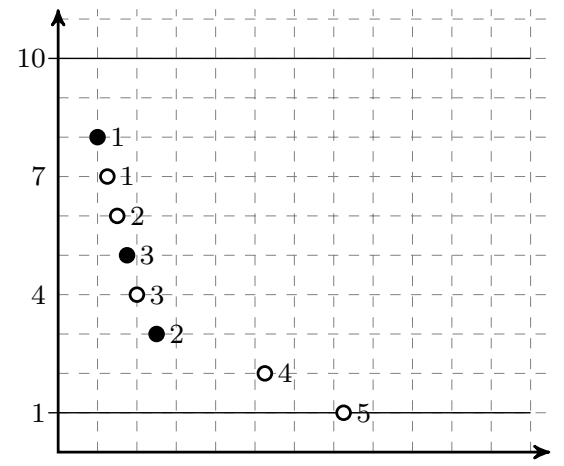

(d)

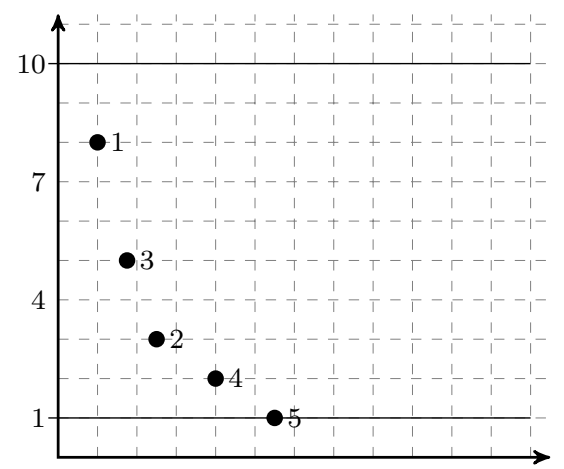

(f)

Figure 4: Possible order in which points of a lower bound set are identified by a sequential search approach. 
Figure ??. There are no converged points at the start of this iteration. In step $I$, seven points are generated. Out of these points, only one of them $(1.0,8.0)$ corresponding to $\operatorname{RLPM}(10)$ is proven to have converged in step $I I$. For the rest of the algorithm, RLPM(10), RLPM(9), and RLPM(8) will be skipped since the first condition for skipping a value of $\varepsilon$ is satisfied for these values.

Figure ??. This iteration starts from $\operatorname{RLPM}(7)$ and generates seven points in step $I$ out of which only one is proven to have converged in step $I I$. The converged point is $(2.5,3.0)$ and corresponds to $\operatorname{RLPM}(3)$.

Figure ??. The two points that converged in the preceding iterations are known at the beginning of this iteration. Step $I$ starts from $\operatorname{RLPM}(7)$ and generates six points and one of these points, $(1.75,5.0)$ corresponding to $\operatorname{RLPM}(5)$, is proven to have converged in step $I I$. It is noted that the second condition for skipping a value of $\varepsilon$ is satisfied for the first three unconverged points. For this reason, $\operatorname{RLPM}(7), \operatorname{RLPM}(6)$, and $\operatorname{RLPM}(4)$ will be skipped for the rest of the algorithm.

Figure ??. Step $I$ produces two points $(4.0,2.0)$ and $(6.5,1.0)$ corresponding to $\operatorname{RLPM}(2)$ and $\operatorname{RLPM}(1)$, respectively. In step $I I$, the point $(4.0,2.0)$ is proven to have converged whereas the other point remains unconverged.

Figure ??. RLPM(1) is solved by column generation until it converges to the point $(5.5,1.0)$. Note that this is the same as repeating steps $I$ and $I I$ the necessary number of times until RLPM(1) converges.

An advantage of the sequential search approach is that, it ensures some uniformity in the convergence of the members of $\boldsymbol{L}$. This can be a very useful technique in the design of heuristics and metaheuristics that are based on column generation since we are sure to find a set of points that is representative of the whole range of the Pareto frontier. Another advantage of the sequential search approach is that it encourages similar columns to appear in the solution of the points in a bound set and this is very necessary in some applications. An example is the case of creating treatment plans in the field of radiotherapy [?]. A bi-objective problem studied in this field is to treat defective cells and also reduce the side effects resulting from the use of radio waves. A column in the model used represents a weekly treatment plan which may require the expertise of several workers. A complete treatment program consists of several weekly treatment plans. Clearly, a treatment program that is made up of too many different weekly plans will be impractical to implement. Applying a sequential search approach to such a problem can help in obtaining more practical plans. 


\section{Application to the Bi-Objective Multi-Vehicle Covering Tour Problem}

In this section, we use the ideas presented in the preceding section to compute lower and upper bound sets for a bi-objective multi-vehicle extension of the covering tour problem (CTP) [?]. This extension is called the bi-objective multivehicle CTP (BOMCTP). We compute a lower bound set by using an $\varepsilon$-constraint

scalarization since we have a condition to efficiently determine possible values of $\varepsilon$.

\subsection{Problem Description}

The CTP consists in designing a route over a subset of locations with the aim of minimizing the length of the route. In addition, each location not visited by the route should lie within a fixed radius from a visited location. The fixed radius is called the cover distance. The CTP has a generic application in the design of bi-level transportation networks [?]. The aim in this kind of problems is to construct a primary route of minimum length in such a way that all points that are not on it can easily reach it. Specific applications arise in the problem of choosing where to locate post boxes among a set of candidate locations [?] and also in the delivery of medical services to villages in developing countries [?, ?]. A bi-objective generalization [?] as well as a multi-vehicle extension [?] of the CTP have been proposed. The cover distance in the bi-objective version is not fixed in advance but rather induced by the constructed route. It is computed by assigning each non-visited location to the closest visited location and calculating the maximum of these distances. The objectives are to minimize the length of the route as well as the induced cover distance. In the multi-vehicle version, the combined length of a set of routes is minimized for a fixed cover distance. In addition, all routes must start from a common location (called the depot) and the number of locations that a single route can visit is limited by a predetermined constant $p$.

The BOMCTP discussed in this paper can be seen as a combination of the bi-objective and the multi-vehicle versions of the CTP and it is defined on a graph $G=(V \cup W, E)$. The nodes of $V$ represent locations which may be visited by a route whereas the members of $W$ are to be assigned to visited nodes of $V$. There is a subset of nodes $T \subseteq V$ which must be visited by at least one route. In particular, $v_{0} \in T$ is the depot where all routes must start and also end. Set $E$ consist of edges connecting all pairs of nodes in $V \cup W$ and a distance matrix $D=\left(d_{i j}\right)$ satisfying the triangle inequality is defined on this set. The BOMCTP consists in designing a set of routes over a subset of $V$ which should include all nodes of $T$ and such that each route visits not more than $p$ nodes of $V \backslash\left\{v_{0}\right\}$. The two objectives are to minimize the total length of the set of routes and the cover distance induced by the set. 


\subsection{A Set Covering Formulation for the BOMCTP}

Let $\Omega$ represent the set of all feasible routes. A feasible route is defined as a Hamiltonian cycle over a subset of $V$ which includes the depot and visits not more than $p$ nodes. The cost of a route $k \in \Omega$ is given by the sum of the cost of the edges it uses and we denote it by $c_{k}$. Let variable $\theta_{k}=1$ if route $k$ is selected in the solution and $\theta_{k}=0$, otherwise. Constant $a_{i k}=1$ if route $k$ visits node $v_{i} \in V \backslash\left\{v_{0}\right\}$ and $a_{i k}=0$ if this is not the case. Variable $z_{i j}$ is used to indicate whether node $w_{j} \in W$ is assigned to $v_{i} \in V \backslash\left\{v_{0}\right\}$ in the solution $\left(z_{i j}=1\right)$ or not $\left(z_{i j}=0\right)$. Let $\Gamma_{\max }$ be the cover distance induced by a given set of routes. Note that $\Gamma_{\max }=\max \left\{d_{i j} z_{i j}: v_{i} \in V \backslash\left\{v_{0}\right\}\right.$ and $\left.w_{j} \in W\right\}$. The BOMCTP can be described with the following set covering model.

$$
\begin{aligned}
\text { Minimize } & \sum_{k \in \Omega} c_{k} \theta_{k} \\
\text { Minimize } & \Gamma_{\max } \\
\text { subject to : } \quad d_{\text {max }}-d_{i j} z_{i j} \geq 0 & \left(v_{i} \in V \backslash\left\{v_{0}\right\}, w_{j} \in W\right), \\
\sum_{v_{i} \in V \backslash\left\{v_{0}\right\}} z_{i j} \geq 1 & \left(w_{j} \in W\right), \\
\sum_{k \in \Omega} a_{i k} \theta_{k}-z_{i j} \geq 0 & \left(v_{i} \in V \backslash\left\{v_{0}\right\}, w_{j} \in W\right), \\
\sum_{k \in \Omega} a_{i k} \theta_{k} \geq 1 & \left(v_{i} \in T \backslash\left\{v_{0}\right\}\right), \\
\Gamma_{\max } \geq 0, & \\
z_{i j} \in\{0,1\} & \left(v_{i} \in V \backslash\left\{v_{0}\right\}, w_{j} \in W\right), \\
\theta_{k} \in \mathbb{N} & (k \in \Omega) .
\end{aligned}
$$

In this formulation, the objectives of minimizing the total length of the set of routes and the induced cover distance are represented in (??) and (??), respectively. Constraints (??) indicate that the induced cover distance should be large enough to respect the distances of all assignments of a node $w_{j} \in W$ to a node $v_{i} \in V \backslash\left\{v_{0}\right\}$. Constraints (??) and (??) specify that each node of $W$ should be assigned to at least one node of $V \backslash\left\{v_{0}\right\}$ which is visited by a route selected in the solution. The requirement that each node of $T \backslash\left\{v_{0}\right\}$ is visited by at least one selected route is represented in Constraints (??). Constraints (??-??) define the domains of the decision variables used. We define $\theta_{k}$ to be a non-negative integer instead of binary in order to prevent constraints of the form $\theta_{k} \leq 1$ in the linear relaxation. The optimal solution of the problem is not affected by this change. 


\subsection{Restricted Linear Programming Master Problem}

We restrict the value of the cover distance induced by the set of routes to be at most $\varepsilon$ to obtain the constraint

$$
-\Gamma_{\max } \geq-\varepsilon
$$

The LPM is defined by the linear relaxation of the formulation with objective function (??) and Constraints (??-??). The RLPM is obtained by replacing $\Omega$ in the LPM with a subset $\Omega_{1}$ for which the problem is primal feasible.

Clearly, there is a finite number of values for $\Gamma_{\max }$ in the range $[\min \varepsilon, \max \varepsilon]$. The value for $\min \varepsilon$ can be obtained when all nodes of $V \backslash\left\{v_{0}\right\}$ are visited by a route so that each node of $W$ can be assigned to the node of $V \backslash\left\{v_{0}\right\}$ which is

closest to it. In a similar way, a value for $\max \varepsilon$ can be obtained by constructing a single route of the form $v_{0} \rightarrow v_{i} \rightarrow v_{0}$ where $v_{i}$ is the closest node of $V \backslash\left\{v_{0}\right\}$ to the depot, $v_{0}$. In such a situation, $v_{i}$ will be required to cover all the nodes of $W$ and so induce a maximum reasonable value for $\Gamma_{\max }$. From the definition of the induced cover distance $\left(\Gamma_{\max }\right)$, the value of $d_{i j}$ for every couple $\left(v_{i}, w_{j}\right) \in V \backslash\left\{v_{0}\right\} \times W$ is a candidate value for $\Gamma_{\max }$. Nevertheless, some of these candidate values will never be the cover distance. We use an idea introduced in [?] and which we state in the next proposition to determine the values that can be the cover distance for any given instance.

Proposition 3.1. Given $v_{i} \in V \backslash\left\{v_{0}\right\}$ and $w_{j} \in W, d_{i j}$ is a feasible value for $\Gamma_{\max }$ if and only if the following two conditions are satisfied.

1. $\forall v_{t} \in T \backslash\left\{v_{0}\right\}$ such that $v_{t} \neq v_{i}, d_{i j} \leq d_{t j}$ and

2. $\forall w_{l} \in W$ such that $w_{l} \neq w_{j}, \exists v_{h} \in V \backslash\left\{v_{0}\right\}$ such that $d_{h l} \leq d_{i j} \leq d_{h j}$.

Proof. The proof is given in two parts. Part I shows that if $d_{i j}$ is a feasible value for $\Gamma_{\max }$ then conditions (??) and (??) are satisfied. Part II also shows that if conditions (??) and (??) are satisfied then $d_{i j}$ is a feasible value for $\Gamma_{\max }$.

Part I. The necessary conditions for $d_{i j}$ to be the value of $\Gamma_{\max }$ are that $v_{i}$ is visited by a selected route and $w_{j}$ is assigned to $v_{i}$. Yet, each node of $W$ is assigned to a visited node of $V \backslash\left\{v_{0}\right\}$ that is closest to it. Since all nodes $v_{t} \in T \backslash\left\{v_{0}\right\}$ are visited in any feasible solution, $w_{j}$ will be assigned to $v_{i}$ only if $d_{i j} \leq d_{t j}$. This proves condition (??). Next, suppose that $\Gamma_{\max }=d_{i j}$ but condition (??) is false. That is, there exists $w_{l} \in W$ such that $w_{l} \neq w_{j}$ and either $d_{i j}>d_{h j}$ or $d_{i j}<d_{h l}$ for all $v_{h} \in V \backslash\left\{v_{0}\right\}$. Then, either $w_{j}$ will be assigned to $v_{h}$ (since $v_{h}$ is closer to it than $v_{i}$ ) or if $w_{j}$ is assigned to $v_{i}$ then $d_{h l}$ is a better candidate for $\Gamma_{\max }$ (since it is greater than $d_{i j}$ and $\Gamma_{\max }$ is determined by the maximum of the assigned distances). In both cases, $d_{i j}$ cannot be the value for $\Gamma_{\max }$ and so condition (??) must be true if $\Gamma_{\max }=d_{i j}$.

Part II. If conditions (??) and (??) are satisfied then $w_{j}$ will be assigned to $v_{i}$ (if it is visited) rather than to a node $v_{t} \in T \backslash\left\{v_{0}\right\}$. If $v_{i}$ is visited and $w_{j}$ is assigned to $v_{i}$, then condition (??) implies that $d_{i j}$ can possibly be the maximum value among all assigned distances and so a feasible value for $\Gamma_{\max }$. 
Using the two conditions in Proposition ??, all the feasible values of $\Gamma_{\max }$ can be computed for any given instance of the BOMCTP. Thus, instead of fixing $\varepsilon$ to the next integer value less than the value of $\Gamma_{\max }$ obtained in a previous step, we rather fix it to the next feasible value of $\Gamma_{\max }$. By doing so, some unnecessary iterations can be avoided during the computation of the set of lower bounds.

\subsection{Dual of LPM}

Let $\gamma_{i j}\left(v_{i} \in V \backslash\left\{v_{0}\right\}, w_{j} \in W\right), \beta_{j}\left(w_{j} \in W\right), \alpha_{i j}\left(v_{i} \in V \backslash\left\{v_{0}\right\}, w_{j} \in W\right)$, $\pi_{i}\left(v_{i} \in T \backslash\left\{v_{0}\right\}\right)$ and $\lambda$ be the non-negative dual variables associated with Constraints (??), (??), (??), (??) and (??), respectively. The dual formulation of the LPM is given by:

$$
\operatorname{maximize} \sum_{w_{j} \in W} \beta_{j}+\sum_{v_{i} \in T \backslash\left\{v_{0}\right\}} \pi_{i}-\varepsilon \lambda
$$

subject to :

$$
\begin{array}{rlrl}
\sum_{\substack{v_{i} \in V \backslash\left\{v_{0}\right\} \\
w_{j} \in W}} a_{i k} \alpha_{i j}+\sum_{v_{i} \in T \backslash\left\{v_{0}\right\}} a_{i k} \pi_{i} \leq c_{k} & & (k \in \Omega), \\
\beta_{j}-d_{i j} \gamma_{i j}-\alpha_{i j} \leq 0 & & \left(v_{i} \in V \backslash\left\{v_{0}\right\}, w_{j} \in W\right), \\
\sum_{\substack{v_{i} \in V \backslash\left\{v_{0}\right\} \\
w_{j} \in W}} \gamma_{i j}-\lambda \leq 0, & \\
\gamma_{i j} \geq 0 & \left(v_{i} \in V \backslash\left\{v_{0}\right\}, w_{j} \in W\right), \\
\alpha_{i j} \geq 0 & \left(v_{i} \in V \backslash\left\{v_{0}\right\}, w_{j} \in W\right), \\
\beta_{j} \geq 0 & \left(w_{j} \in W\right), \\
\pi_{i} \geq 0 & \left(v_{i} \in T \backslash\left\{v_{0}\right\}\right), \\
\lambda \geq 0 . &
\end{array}
$$

\subsection{Sub-problem}

The sub-problem is to search for feasible routes $k \in \Omega \backslash \Omega_{1}\left(\Omega_{1}\right.$ is the set of columns in the RLPM at any given time) such that

$$
c_{k}-\sum_{\substack{v_{i} \in V \backslash\left\{v_{0}\right\} \\ w_{j} \in W}} a_{i k} \alpha_{i j}-\sum_{v_{i} \in T \backslash\left\{v_{0}\right\}} a_{i k} \pi_{i}<0 .
$$

We define $\pi_{i}^{*}=\pi_{i}$ if $v_{i} \in T \backslash\left\{v_{0}\right\}$ and $\alpha_{i j}^{*}=\alpha_{i j}$ if $v_{i} \in V \backslash\left\{v_{0}\right\}, w_{j} \in W$. In all other cases these variables are set to 0 . We also let $x_{i j k}=1$ if route $k$ visits $v_{j}$ immediately after visiting $v_{i}$ and $x_{i j k}=0$ if this is not the case.

Note that $c_{k}=\sum_{\left(v_{i}, v_{j}\right) \in E} x_{i j k} d_{i j}$ and $\sum_{v_{i} \in V \backslash\left\{v_{0}\right\}} a_{i k}=\sum_{v_{i} \in V} a_{i k}=\sum_{\left(v_{i}, v_{j}\right) \in E} x_{i j k}$. 
The starred versions of the dual variables $\left(\alpha_{i j}^{*}\right.$ and $\left.\pi_{i}^{*}\right)$ also allow us to write the summations over $V \backslash\left\{v_{0}\right\}$ and $T \backslash\left\{v_{0}\right\}$ as a summation over $V$. We get

$$
\begin{gathered}
\sum_{\substack{v_{i} \in V \backslash\left\{v_{0}\right\} \\
w_{j} \in W}} a_{i k} \alpha_{i j}=\sum_{v_{i} \in V}\left(\sum_{w_{h} \in W} \alpha_{i h}^{*}\right) a_{i k}=\sum_{\left(v_{i}, v_{j}\right) \in E}\left(\sum_{w_{h} \in W} \alpha_{i h}^{*}\right) x_{i j k} \\
\text { and } \sum_{v_{i} \in T \backslash\left\{v_{0}\right\}} a_{i k} \pi_{i}=\sum_{v_{i} \in V} a_{i k} \pi_{i}^{*}=\sum_{\left(v_{i}, v_{j}\right) \in E} \pi_{i}^{*} x_{i j k} .
\end{gathered}
$$

Condition (??) can, thus, be simplified to

$$
\sum_{\left(v_{i}, v_{j}\right) \in E}\left(d_{i j}-\pi_{i}^{*}-\sum_{w_{h} \in W} \alpha_{i h}^{*}\right) x_{i j k}<0 .
$$

Feasible routes satisfying condition (??) are searched for by solving an elementary shortest path problem with resource constraints (ESPPRC) given by

$$
\text { Minimize } \sum_{\left(v_{i}, v_{j}\right) \in E}\left(d_{i j}-\pi_{i}^{*}-\sum_{w_{h} \in W} \alpha_{i h}^{*}\right) x_{i j k} \quad \text { subject to } k \in \Omega \backslash \Omega_{1} .
$$

That is, we seek feasible routes with negative reduced costs where the reduced cost of $\left(v_{i}, v_{j}\right) \in V \times V$ is given by $d_{i j}-\pi_{i}^{*}-\sum_{w_{h} \in W} \alpha_{i h}^{*}$. The ESPPRC is very well studied since it appears as a subproblem in many vehicle routing problems solved by column generation. The problem is $\mathcal{N} \mathcal{P}$-hard in the strong sense [?]. Several algorithms based on dynamic programming have been proposed to solve the ESPPRC. These algorithms rely mainly on the label setting algorithm for the shortest path problem with resource constraint (SPPRC) [?] where a route may visit a given node more than once. An exact algorithm for the ESPPRC is given in [?]. We solve the subproblem by the decremental state space relaxation (DSSR) algorithm [?, ?]. The only resource constraint considered is the limit on the total number of nodes a route can visit.

\section{Computational Experiments}

We have performed numerical experiments to evaluate the quality of lower and upper bound sets for the BOMCTP and also compare the relative performance of the different column search approaches. In order to evaluate the quality of a lower bound set and a corresponding upper bound set, we used a distance based measure $\left(\mu_{1}\right)$, and an area based measure $\left(\mu_{2}\right)$ which were presented in [?]. Combining an area based measure with a distance based measure gives a good indication of the quality of the bounds. Roughly speaking, $\mu_{1}$ represents the worst distance (with respect to the range of objective values) between a point of the upper bound set and a point of lower bound set closest to it. Also, $\mu_{2}$ represents the fraction of the area that is dominated by the lower bound set but not by the upper bound set. This is, the area where additional points of $\mathcal{Y}_{N}$ can 
be found. If a lower bound set and a corresponding upper bound set are good, then we expect that both $\mu_{1}$ and $\mu_{2}$ will be small in value. The smaller both values are, the better the quality of the bounds. These two measures complement each other and as explained by the authors of [?], the measures can be seen to play a role analogous to the optimality gap in single objective optimization. The reader is referred to the relevant paper [?] for further explanation of the measures.

Before presenting the results obtained for the BOMCTP, we first give an overview of the quality of lower bound sets obtained by the weighted sum and the $\varepsilon$-constraint methods for the bi-objective set covering problem.

\subsection{Quality of Lower Bound Sets for the Bi-Objective Set Covering Problem}

We compare the quality of lower bound sets computed by the weighted sum and $\varepsilon$-constraint methods for the bi-objective set covering problem (BOSCP). Since the BOSCP serves as a base formulation for most problems solved by column generation, the aim of this section is to give a general idea of the quality of lower bounds computed by the two scalarization methods for this kind of problem and its extensions. The BOSCP is formulated as :

$$
\begin{aligned}
\text { Minimize } & \sum_{j=1}^{n} c_{j}^{1} x_{j} \\
\text { Minimize } & \sum_{j=1}^{n} c_{j}^{2} x_{j} \\
\text { subject to : } & \sum_{j=1}^{n} a_{i j} x_{j} \geq 1 \quad i=1,2, \ldots, m, \\
& x_{j} \in\{0,1\} \quad j=1,2, \ldots, n .
\end{aligned}
$$

The integral objective coefficients are $c_{j}^{1}$ and $c_{j}^{2}$. The matrix coefficients are $a_{i j} \in\{0,1\}$ and we say that constraint $i$ is covered by variable $x_{j}$ if $a_{i j}=1$. The BOSCP instances used for the tests can be found at http://xgandibleux. free.fr/MOCOlib/MOSCP.html. They are the same instances that were used in [?]. Since the goal is to evaluate the quality of lower bound sets, we used the exact nondominated sets computed by the algorithm proposed in [?] as upper bound sets. For this reason, the values of the two quality measures obtained for the weighted sum method are different from those obtained in [?] when greedy heuristics are used to compute upper bound sets. All computer codes were written in $\mathrm{C} / \mathrm{C}++$ and the LP/MIP optimizers of ILOG CPLEX 12.4 were used. The tests were run on an Intel(R) Core(TM)2 Duo CPU E7500 @ $2.93 \mathrm{GHz}$ computer with a $2 \mathrm{GiB}$ RAM and a summary of the results are presented in Table ??. In this table, $\left|\boldsymbol{U}^{*}\right|$ represents the number of points in the exact nondominated set, time is the computational time in cpu seconds, $|e x t|$ is the number of extreme points computed when the weighted sum method is used, 
Table 1: Comparison of lower bound sets for the BOSCP

\begin{tabular}{|c|c|c|c|c|c|c|c|c|c|}
\hline \multirow[b]{2}{*}{ Instance } & \multirow[b]{2}{*}{$\left|\boldsymbol{U}^{*}\right|$} & \multicolumn{4}{|c|}{ Weighted Sum } & \multicolumn{4}{|c|}{$\varepsilon$-Constraint } \\
\hline & & time & $|e x t|$ & $\mu_{1} \%$ & $\mu_{2} \%$ & time & $|\boldsymbol{L}|$ & $\mu_{1} \%$ & $\mu_{2} \%$ \\
\hline $2 \mathrm{scp} 11 \mathrm{~A}$ & 39 & 0.00 & 13 & 2.9 & 2.5 & 0.02 & 192 & 2.7 & 2.1 \\
\hline $2 \mathrm{scp} 11 \mathrm{C}$ & 10 & 0.00 & 21 & 13.7 & 34.1 & 0.01 & 117 & 13.9 & 32.7 \\
\hline $2 \operatorname{scp} 41 \mathrm{~A}$ & 105 & 0.01 & 23 & 1.5 & 1.3 & 0.14 & 1028 & 1.4 & 1.2 \\
\hline $2 \mathrm{scp} 41 \mathrm{C}$ & 24 & 0.02 & 17 & 2.6 & 4.5 & 0.04 & 280 & 2.7 & 5.7 \\
\hline $2 \operatorname{scp} 42 A$ & 206 & 0.02 & 37 & 0.6 & 0.5 & 0.45 & 1663 & 0.5 & 0.4 \\
\hline $2 \mathrm{scp} 42 \mathrm{C}$ & 87 & 0.04 & 51 & 3.0 & 3.4 & 0.44 & 2245 & 2.9 & 3.4 \\
\hline $2 \mathrm{scp} 43 \mathrm{~A}$ & 46 & 0.04 & 63 & 3.6 & 4.3 & 0.12 & 473 & 3.5 & 4.1 \\
\hline $2 \mathrm{scp} 43 \mathrm{C}$ & 12 & 0.02 & 38 & 6.3 & 11.1 & 0.04 & 225 & 6.3 & 10.6 \\
\hline $2 \mathrm{scp} 61 \mathrm{~A}$ & 254 & 0.06 & 58 & 1.1 & 0.8 & 1.39 & 2829 & 1.1 & 0.8 \\
\hline $2 \mathrm{scp} 61 \mathrm{C}$ & 28 & 0.02 & 17 & 1.0 & 3.7 & 1.02 & 732 & 0.9 & 4.4 \\
\hline $2 \mathrm{scp} 62 \mathrm{~A}$ & 98 & 0.28 & 236 & 3.5 & 3.6 & 1.01 & 1240 & 3.5 & 3.7 \\
\hline $2 \mathrm{scp} 62 \mathrm{C}$ & 6 & 0.23 & 180 & 38.9 & 46.6 & 0.00 & 2 & 36.6 & 58.5 \\
\hline $2 \operatorname{scp} 81 \mathrm{~A}$ & 424 & 0.07 & 47 & 0.5 & 0.4 & 3.49 & 5580 & 0.4 & 0.3 \\
\hline $2 \mathrm{scp} 81 \mathrm{C}$ & 14 & 0.12 & 77 & 0.3 & 0.5 & 0.60 & 147 & 0.4 & 0.7 \\
\hline
\end{tabular}

and $|\boldsymbol{L}|$ is the total number of points in a lower bound set when an $\varepsilon$-constraint method is used. Values of the two quality measures are expressed as percentages under the headings $\mu_{1} \%$ and $\mu_{2} \%$.

The results show that, the lower bound sets obtained by both methods for almost all the instances are of good quality. The lower bounds obtained in the case of the $\varepsilon$-constraint method are slightly better than those obtained for the weighted sum method. The situation is quite different when we compare the computational times and the computational complexities of the two methods. In the case of the weighted sum method, relatively few extreme points need to be calculated and this is done in negligible time for the instances tested. The number of points that needs to be calculated when an $\varepsilon$-constraint method is used can be very large (possibly exponential) and this may require a very long computational time. For example, the $\varepsilon$-constraint method computed 5580 points in a time of $3.49 \mathrm{cpu}$ seconds for instance $2 \mathrm{scp} 81 \mathrm{~A}$. This is very large when we compare it with the 47 points computed in 0.07 seconds when a weighted sum method was used. This stresses the need to reduce the number of points calculated when using an $\varepsilon$-constraint method by determining good step sizes.

\subsection{Experiments for the BOMCTP}

We now present results from experiments conducted to evaluate the quality of lower and upper bound sets computed for the BOMCTP. We also compare the relative perfomance of the different column search approaches. 
Table 2: Quality of bound sets for the BOMCTP $(p=5)$

\begin{tabular}{|c|c|c|c|c|c|c|c|c|c|c|c|c|}
\hline \multirow[b]{2}{*}{$|T|$} & \multirow[b]{2}{*}{$|V|$} & \multirow[b]{2}{*}{$|W|$} & \multirow[b]{2}{*}{$\left|\boldsymbol{L}^{*}\right|$} & \multicolumn{3}{|c|}{ PPS } & \multicolumn{3}{|c|}{$k$-PPS } & \multicolumn{3}{|c|}{ Sequential } \\
\hline & & & & $|\boldsymbol{U}|$ & $\mu_{1} \%$ & $\mu_{2} \%$ & $|\boldsymbol{U}|$ & $\mu_{1} \%$ & $\mu_{2} \%$ & $|\boldsymbol{U}|$ & $\mu_{1} \%$ & $\mu_{2} \%$ \\
\hline 1 & 30 & 60 & 64 & 29 & 5.2 & 26.2 & 28 & 5.2 & 26.1 & 29 & 5.1 & 26.0 \\
\hline 1 & 30 & 90 & 66 & 29 & 6.0 & 27.6 & 29 & 6.1 & 27.5 & 28 & 5.9 & 27.5 \\
\hline 1 & 40 & 80 & 58 & 30 & 5.5 & 26.1 & 29 & 5.6 & 26.0 & 30 & 5.4 & 25.9 \\
\hline 1 & 40 & 120 & 67 & 37 & 5.1 & 25.4 & 29 & 5.1 & 25.4 & 36 & 5.0 & 25.2 \\
\hline 1 & 50 & 100 & 65 & 34 & 4.9 & 24.7 & 34 & 4.9 & 24.5 & 35 & 4.8 & 24.5 \\
\hline 1 & 50 & 150 & 67 & 35 & 4.5 & 24.8 & 39 & 4.6 & 24.7 & 36 & 4.5 & 24.0 \\
\hline 8 & 30 & 60 & 15 & 9 & 7.7 & 54.1 & 9 & 7.7 & 54.1 & 9 & 7.6 & 54.1 \\
\hline 8 & 30 & 90 & 17 & 10 & 11.0 & 53.3 & 10 & 11.1 & 53.3 & 10 & 11.0 & 53.2 \\
\hline 10 & 40 & 80 & 15 & 11 & 1.4 & 58.1 & 11 & 1.4 & 58.1 & 12 & 1.3 & 58.1 \\
\hline 10 & 40 & 120 & 16 & 13 & 9.1 & 61.7 & 12 & 9.1 & 61.7 & 14 & 9.0 & 61.8 \\
\hline 13 & 50 & 100 & 18 & 11 & 16.7 & 72.9 & 11 & 16.7 & 80.0 & 11 & 16.6 & 72.7 \\
\hline 13 & 50 & 150 & 19 & 10 & 21.6 & 72.6 & 11 & 21.5 & 72.7 & 12 & 21.6 & 72.9 \\
\hline 15 & 30 & 60 & 2 & 6 & 20.3 & 54.7 & 5 & 20.3 & 54.6 & 6 & 20.3 & 54.6 \\
\hline 15 & 30 & 90 & 5 & 6 & 40.5 & 55.7 & 6 & 40.5 & 55.7 & 6 & 40.4 & 55.7 \\
\hline 20 & 40 & 80 & 6 & 4 & 57.3 & 61.4 & 5 & 57.3 & 61.4 & 5 & 57.3 & 61.4 \\
\hline 20 & 40 & 120 & 7 & 5 & 31.2 & 62.3 & 6 & 31.2 & 62.3 & 6 & 31.2 & 62.3 \\
\hline 25 & 50 & 100 & 6 & 4 & 24.3 & 76.8 & 4 & 24.3 & 76.5 & 4 & 24.3 & 76.8 \\
\hline 25 & 50 & 150 & 9 & 4 & 28.9 & 79.1 & 4 & 28.9 & 78.9 & 5 & 28.9 & 79.0 \\
\hline
\end{tabular}

\subsubsection{Description of Instances and Experiments}

The Mersenne Twister random number generator was used to generate instances similar to those described in the literature [?, ?, ?] but which are not publicly available. The generator can be obtained at http://www.math.sci . hiroshima-u.ac.jp/ m-mat/MT/emt.html. The node sets were obtained by generating $|V|+|W|$ points in the $[0,100] \times[0,100]$ square with the depot restricted to lie in $[25,75] \times[25,75]$. Set $T$ (respectively, $V$ ) is taken to be the first $|T|$ (respectively, $|V|$ ) points and set $W$ is taken as the remaining points. The distance between two points is calculated as the Euclidean distance between them. Five instances for every combination of $|V| \in\{30,40,50\}$ and $|W| \in\{2|V|, 3|V|\}$ were generated. Values of $|T|$ in $\{1,\lceil 0.25|V|\rceil,\lceil 0.50|V|\rceil\}$ and $p$ in $\{5,8\}$ were tested. The exact instances used for our experiments can be found at http://homepages.laas.fr/bmsarpon/ctp_instances.zip. All computer codes were written in $\mathrm{C} / \mathrm{C}++$ and the RMP was solved with ILOG CPLEX 12.4. Tests were run on an Intel(R) Core(TM)2 Duo CPU E7500 @ $2.93 \mathrm{GHz}$ computer with a $2 \mathrm{GiB}$ RAM. Summary of the results are given in Tables ?? to ??. All the values in these tables are averages over the five generated instances.

The condition we used in implementing the $k$-PPS, is to skip a current value of $\varepsilon$ when the objective value of the RLPM has not improved (i.e. decreased) 
Table 3: Quality of bound sets for the BOMCTP $(p=8)$

\begin{tabular}{|c|c|c|c|c|c|c|c|c|c|c|c|c|}
\hline \multirow[b]{2}{*}{$|T|$} & \multirow[b]{2}{*}{$|V|$} & \multirow[b]{2}{*}{$|W|$} & \multirow[b]{2}{*}{$\left|\boldsymbol{L}^{*}\right|$} & \multicolumn{3}{|c|}{ PPS } & \multicolumn{3}{|c|}{$k$-PPS } & \multicolumn{3}{|c|}{ Sequential } \\
\hline & & & & $|\boldsymbol{U}|$ & $\mu_{1} \%$ & $\mu_{2} \%$ & $|\boldsymbol{U}|$ & $\mu_{1} \%$ & $\mu_{2} \%$ & $|\boldsymbol{U}|$ & $\mu_{1} \%$ & $\mu_{2} \%$ \\
\hline 1 & 30 & 60 & 64 & 29 & 5.3 & 26.3 & 29 & 5.3 & 26.1 & 30 & 5.2 & 26.2 \\
\hline 1 & 30 & 90 & 63 & 30 & 6.5 & 28.2 & 29 & 6.5 & 28.1 & 29 & 6.4 & 28.0 \\
\hline 1 & 40 & 80 & 58 & 28 & 5.6 & 26.0 & 28 & 5.5 & 26.0 & 30 & 5.6 & 26.0 \\
\hline 1 & 40 & 120 & 65 & 35 & 5.2 & 25.7 & 26 & 5.3 & 25.4 & 36 & 5.1 & 25.2 \\
\hline 1 & 50 & 100 & 65 & 34 & 4.9 & 24.7 & 34 & 4.9 & 24.6 & 33 & 4.7 & 24.6 \\
\hline 1 & 50 & 150 & 64 & 36 & 4.7 & 24.8 & 38 & 4.6 & 24.5 & 36 & 4.7 & 24.4 \\
\hline 8 & 30 & 60 & 15 & 10 & 10.2 & 65.1 & 10 & 10.2 & 65.0 & 10 & 10.2 & 65.1 \\
\hline 8 & 30 & 90 & 15 & 9 & 28.7 & 69.5 & 10 & 26.8 & 69.7 & 10 & 20.3 & 69.6 \\
\hline 10 & 40 & 80 & 14 & 8 & 16.6 & 68.0 & 8 & 16.6 & 67.9 & 9 & 16.6 & 67.8 \\
\hline 10 & 40 & 120 & 17 & 11 & 9.3 & 73.4 & 10 & 9.3 & 73.3 & 11 & 9.2 & 73.3 \\
\hline 13 & 50 & 100 & 16 & 10 & 35.2 & 74.2 & 10 & 35.2 & 74.2 & 10 & 35.1 & 74.2 \\
\hline 13 & 50 & 150 & 17 & 9 & 30.1 & 76.7 & 9 & 30.1 & 76.7 & 10 & 30.1 & 76.7 \\
\hline 15 & 30 & 60 & 2 & 6 & 29.0 & 65.4 & 6 & 29.0 & 65.4 & 6 & 29.0 & 65.4 \\
\hline 15 & 30 & 90 & 2 & 6 & 35.4 & 71.1 & 6 & 35.4 & 71.3 & 6 & 35.4 & 71.0 \\
\hline 20 & 40 & 80 & 2 & 4 & 41.2 & 72.9 & 5 & 41.3 & 73.0 & 5 & 41.1 & 72.7 \\
\hline 20 & 40 & 120 & 4 & 6 & 9.2 & 73.8 & 6 & 9.1 & 73.8 & 6 & 9.1 & 73.2 \\
\hline 25 & 50 & 100 & 3 & 5 & 34.9 & 81.3 & 5 & 35.0 & 81.1 & 6 & 34.9 & 81.0 \\
\hline 25 & 50 & 150 & 4 & 5 & 24.3 & 87.9 & 6 & 24.2 & 87.3 & 7 & 24.2 & 87.2 \\
\hline
\end{tabular}

by more than 1 after $k=3$ iterations of column generation. Given that column generation is an exact method for the RMP, the same lower bound set is obtained for a given instance irrespective of the column search approach used. The number of elements in the common lower bound set for each instance is given under the column with heading $\left|\boldsymbol{L}^{*}\right|$. The number of elements in an upper bound set is given under the columns with heading $|\boldsymbol{U}|$. We recall that for each approach, an upper bound set is computed after computing a lower bound set by following the idea of PPS. The only difference is that we solve integer programs rather than linear relaxations. For each instance, these values are different for the different column search approaches since different columns are generated by each approach when computing a lower bound set. The columns with headings $d s s r$ and cols give the number of times the sub-problem was solved with the DSSR algorithm and the total number of columns generated, respectively, when computing a lower bound set. All the other column headings have the same meanings as those for the BOSCP in Table ??.

\subsubsection{Discussion of Results}

A first general comment is that, the number of points in a bound set decreases when the size of $T$ increases. This can be seen in Tables ?? and ?? under 
Table 4: Computational times for the BOMCTP $(p=5)$

\begin{tabular}{|c|c|c|c|c|c|c|c|c|c|c|c|}
\hline \multirow[b]{2}{*}{$|T|$} & \multirow[b]{2}{*}{$|V|$} & \multirow[b]{2}{*}{$|W|$} & \multicolumn{3}{|c|}{ PPS } & \multicolumn{3}{|c|}{$k$-PPS } & \multicolumn{3}{|c|}{ Sequential } \\
\hline & & & time & dssr & cols & time & dssr & cols & time & dssr & cols \\
\hline 1 & 30 & 60 & 7.78 & 160 & 358 & 6.72 & 126 & 388 & 7.00 & 98 & 372 \\
\hline 1 & 30 & 90 & 12.81 & 93 & 332 & 10.82 & 95 & 364 & 9.13 & 73 & 351 \\
\hline 1 & 40 & 80 & 14.99 & 72 & 416 & 11.37 & 70 & 479 & 7.83 & 59 & 461 \\
\hline 1 & 40 & 120 & 15.98 & 129 & 435 & 12.51 & 112 & 521 & 7.76 & 90 & 478 \\
\hline 1 & 50 & 100 & 25.96 & 152 & 570 & 13.33 & 147 & 574 & 8.81 & 135 & 583 \\
\hline 1 & 50 & 150 & 24.66 & 137 & 591 & 15.29 & 116 & 573 & 11.51 & 106 & 619 \\
\hline 8 & 30 & 60 & 6.32 & 165 & 808 & 8.74 & 147 & 823 & 7.89 & 123 & 747 \\
\hline 8 & 30 & 90 & 15.28 & 143 & 837 & 13.06 & 139 & 891 & 14.14 & 104 & 865 \\
\hline 10 & 40 & 80 & 18.60 & 157 & 969 & 19.37 & 142 & 1036 & 15.60 & 132 & 974 \\
\hline 10 & 40 & 120 & 28.21 & 195 & 1048 & 28.16 & 176 & 986 & 23.92 & 155 & 1096 \\
\hline 13 & 50 & 100 & 42.39 & 243 & 1160 & 39.49 & 198 & 1171 & 36.34 & 174 & 1235 \\
\hline 13 & 50 & 150 & 51.69 & 286 & 1395 & 52.67 & 201 & 1343 & 42.11 & 194 & 1480 \\
\hline 15 & 30 & 60 & 16.81 & 149 & 825 & 15.08 & 126 & 891 & 13.97 & 114 & 926 \\
\hline 15 & 30 & 90 & 26.41 & 153 & 973 & 25.54 & 148 & 974 & 23.78 & 141 & 969 \\
\hline 20 & 40 & 80 & 35.83 & 168 & 1232 & 36.83 & 160 & 1235 & 27.43 & 143 & 1198 \\
\hline 20 & 40 & 120 & 53.45 & 192 & 1568 & 50.74 & 174 & 1549 & 42.81 & 158 & 1480 \\
\hline 25 & 50 & 100 & 55.49 & 227 & 1913 & 49.03 & 195 & 1629 & 44.19 & 172 & 1670 \\
\hline 25 & 50 & 150 & 68.22 & 285 & 2194 & 61.64 & 223 & 2160 & 53.30 & 206 & 2075 \\
\hline
\end{tabular}


Table 5: Computational times for the BOMCTP $(p=8)$

\begin{tabular}{|c|c|c|c|c|c|c|c|c|c|c|c|}
\hline \multirow[b]{2}{*}{$|T|$} & \multirow[b]{2}{*}{$|V|$} & \multirow[b]{2}{*}{$|W|$} & \multicolumn{3}{|c|}{ PPS } & \multicolumn{3}{|c|}{$k$-PPS } & \multicolumn{3}{|c|}{ Sequential } \\
\hline & & & time & dssr & cols & time & dssr & cols & time & dssr & cols \\
\hline 1 & 30 & 60 & 11.82 & 245 & 374 & 15.83 & 183 & 392 & 6.85 & 119 & 449 \\
\hline 1 & 30 & 90 & 14.86 & 144 & 357 & 14.23 & 141 & 383 & 8.33 & 132 & 417 \\
\hline 1 & 40 & 80 & 53.97 & 124 & 436 & 22.24 & 109 & 451 & 17.54 & 93 & 491 \\
\hline 1 & 40 & 120 & 47.20 & 154 & 438 & 29.32 & 127 & 492 & 18.30 & 120 & 524 \\
\hline 1 & 50 & 100 & 96.60 & 189 & 618 & 22.55 & 142 & 575 & 15.09 & 132 & 633 \\
\hline 1 & 50 & 150 & 86.50 & 199 & 566 & 31.13 & 132 & 573 & 19.58 & 111 & 578 \\
\hline 8 & 30 & 60 & 52.13 & 231 & 738 & 53.92 & 211 & 718 & 48.12 & 176 & 619 \\
\hline 8 & 30 & 90 & 58.00 & 195 & 953 & 62.17 & 176 & 949 & 51.30 & 164 & 946 \\
\hline 10 & 40 & 80 & 81.38 & 206 & 1087 & 76.11 & 184 & 1093 & 69.26 & 153 & 1074 \\
\hline 10 & 40 & 120 & 69.48 & 237 & 988 & 61.90 & 201 & 1027 & 54.67 & 159 & 1080 \\
\hline 13 & 50 & 100 & 123.70 & 273 & 1439 & 115.14 & 258 & 1335 & 79.89 & 213 & 1326 \\
\hline 13 & 50 & 150 & 110.41 & 294 & 1712 & 103.65 & 272 & 1726 & 87.10 & 219 & 1766 \\
\hline 15 & 30 & 60 & 42.09 & 259 & 952 & 42.89 & 209 & 968 & 38.16 & 182 & 825 \\
\hline 15 & 30 & 90 & 60.33 & 198 & 1071 & 58.67 & 177 & 1108 & 46.50 & 169 & 1151 \\
\hline 20 & 40 & 80 & 153.70 & 281 & 1209 & 129.85 & 275 & 1160 & 109.25 & 227 & 1080 \\
\hline 20 & 40 & 120 & 118.65 & 296 & 1841 & 103.09 & 278 & 1833 & 98.37 & 254 & 1766 \\
\hline 25 & 50 & 100 & 209.85 & 317 & 2058 & 196.46 & 297 & 2051 & 182.32 & 261 & 2049 \\
\hline 25 & 50 & 150 & 264.91 & 334 & 2314 & 251.92 & 312 & 2217 & 201.49 & 259 & 2147 \\
\hline
\end{tabular}


the column headings $\left|\boldsymbol{L}^{*}\right|$ and $|\boldsymbol{U}|$. From these two tables, the quality of the bound sets obtained are quite good. There seem to be no clear preference for one column search approach with respect to another in terms of the quality of the bound sets obtained. Nevertheless, the quality of bound sets obtained by the sequential search approach are slightly better than those obtained by the PPS and $k$-PPS in most cases. This can be seen, for example, in row $|T|=1$, $|V|=40,|W|=120$ of Table ??. The value of the pair $\left(\mu_{1} \%, \mu_{2} \%\right)$ for the PPS, the $k$-PPS, and the sequential search approaches are $(5.2,25.7),(5.3,25.4)$, and $(5.1,25.2)$, respectivley.

In terms of computational times (see Tables ?? and ??), the sequential search approach performs the best. The $k$-PPS also performs better than the PPS. For example, in the row for $|T|=13,|V|=50,|W|=150$ of Table ??, the computational time for the PPS is 110.41 , the one for the $k$-PPs is 103.65 and that of the sequential search is 87.10 . From the same row, we can also see that the sequential search approach solves a fewer number of subproblems (219) with respect to the PPS (294) and the $k$-PPS (272). This is a very good sign in favour of the sequential search approach since solving the subproblem is usually the most costly operation in a column generation algorithm.

\section{Conclusions}

This paper discusses the use of column generation in computing bound sets for bi-objective combinatorial optimization problems. The main idea used is to convert the bi-objective problem into single objective by a scalarization method and solve the linear relaxation of the resulting single objective problem several times by varying the necessary parameters. Two very popular scalarization methods (weighted sum and $\varepsilon$-constraint) are studied for this purpose. We saw that the subproblems encountered for both scalarization methods have a similar structure. For this reason, we presented some strategies to effectively search for columns in order to possibly take advantage of the similar subproblems. The $k$-PPS and the sequential search approaches were proposed to address some challenges faced by a rather standard approach (the PPS). The ideas presented are applied to the bi-objective multi-vehicle covering tour problem and the results show that the proposed methodology and column search approaches are effective. In particular, the sequential search method is faster and also solves relatively few number of subproblems when computing a lower bound set. A possible extension of this current work is to test the presented strategies in the case of the weighted sum method and also develop other similar strategies. It will also be interesting to develop column generation based heuristics and metaheuristics that use the idea of the sequential search method. 


\section{References}

[1] Yash P Aneja and KPK Nair. Bicriteria transportation problem. Management Science, 25(1):73-78, 1979.

[2] Jean-François Bérubé, Michel Gendreau, and Jean-Yves Potvin. An exact $\epsilon$-constraint method for bi-objective combinatorial optimization problems: Application to the Traveling Salesman Problem with Profits. European Journal of Operational Research, 194(1):39-50, 2009.

[3] Natashia Boland, John Dethridge, and Irina Dumitrescu. Accelerated label setting algorithms for the elementary resource constrained shortest path problem. Operations Research Letters, 34(1):58-68, 2006.

[4] John R. Current and David A. Schilling. The median tour and maximal covering tour problems: Formulations and heuristics. European Journal of Operational Research, 73(1):114-126, 1994.

[5] Charles Delort and Olivier Spanjaard. Using bound sets in multiobjective optimization: Application to the biobjective binary knapsack problem. In Experimental Algorithms, pages 253-265. Springer, 2010.

[6] Martin Desrochers and François Soumis. A generalized permanent labelling algorithm for the shortest path problem with time windows. INFOR, 26:191$212,1988$.

[7] Moshe Dror. Note on the complexity of shortest path models for column generation in VRPTW. Operations Research, 42(5):977-978, 1994.

[8] Matthias Ehrgott and Xavier Gandibleux. Bound sets for biobjective combinatorial optimization problems. Computers $\&$ Operations Research, 34(9):2674-2694, 2007.

[9] Dominique Feillet, Pierre Dejax, Michel Gendreau, and Cyrille Gueguen. An exact algorithm for the Elementary Shortest Path Problem with Resource Constraints: Application to some vehicle routing problems. Networks, 44(3):216-229, 2004.

[10] Michel Gendreau, Gilbert Laporte, and Frédéric Semet. The Covering Tour Problem. Operations Research, 45(4):568-576, 1997.

[11] Mondher Hachicha, M John Hodgson, Gilbert Laporte, and Frédéric Semet. Heuristics for the multi-vehicle covering tour problem. Computers $\& 3$ Operations Research, 27(1):29-42, 2000.

[12] M. John Hodgson, Gilbert Laporte, and Frédéric Semet. A Covering Tour Model for Planning Mobile Health Care Facilities in SuhumDistrict, Ghama. Journal of Regional Science, 38(4):621-638, 1998. 
[13] Nicolas Jozefowiez, Frédéric Semet, and El-Ghazali Talbi. The bi-objective covering tour problem. Computers \& Operations Research, 34(7):1929-1942, 2007.

[14] Martine Labbé and Gilbert Laporte. Maximizing user convenience and postal service efficiency in post box location. Belgian Journal of Operations Research, Statistics and Computer Science, 26:21-35, 1986.

[15] Giovanni Righini and Matteo Salani. New dynamic programming algorithms for the resource constrained elementary shortest path problem. Networks, $51(3): 155-170,2008$.

[16] Ehsan Salari and Jan Unkelbach. A column-generation-based method for multi-criteria direct aperture optimization. Physics in medicine and biology, 58(3):621-639, 2013.

[17] Francis Sourd and Olivier Spanjaard. A multiobjective branch-and-bound framework: Application to the biobjective spanning tree problem. INFORMS Journal on Computing, 20(3):472-484, 2008.

[18] E.L. Ulungu and J. Teghem. The two phases method: An efficient procedure to solve bi-objective combinatorial optimization problems. Foundations of Computing and Decision Sciences, 20(2):149-165, 1995.

[19] François Vanderbeck. Implementing mixed integer column generation. In Guy Desaulniers, Jacques Desrosiers, and Marius M. Solomon, editors, Column Generation, pages 331-358. Springer, 2005.

[20] Bernardo Villarreal and Mark H. Karwan. Multicriteria integer programming: A (hybrid) dynamic programming recursive approach. Mathematical Programming, 21(1):204-223, 1981. 